ARTICLE

DOI: $10.1038 / s 41467-018-07332-5$

\title{
A new look at effective interactions between microgel particles
}

\author{
Maxime J. Bergman (1) 1, Nicoletta Gnan², Marc Obiols-Rabasa (1) 1,3, Janne-Mieke Meijer (1) 1,4, \\ Lorenzo Rovigatti (i) $^{2}$, Emanuela Zaccarelli (iD ${ }^{2}$ \& Peter Schurtenberger (i) ${ }^{1}$
}

Thermoresponsive microgels find widespread use as colloidal model systems, because their temperature-dependent size allows facile tuning of their volume fraction in situ. However, an interaction potential unifying their behavior across the entire phase diagram is sorely lacking. Here we investigate microgel suspensions in the fluid regime at different volume fractions and temperatures, and in the presence of another population of small microgels, combining confocal microscopy experiments and numerical simulations. We find that effective interactions between microgels are clearly temperature dependent. In addition, microgel mixtures possess an enhanced stability compared to hard colloid mixtures - a property not predicted by a simple Hertzian model. Based on numerical calculations we propose a multi-Hertzian model, which reproduces the experimental behavior for all studied conditions. Our findings highlight that effective interactions between microgels are much more complex than usually assumed, displaying a crucial dependence on temperature and on the internal core-corona architecture of the particles.

\footnotetext{
${ }^{1}$ Division of Physical Chemistry, Department of Chemistry, Lund University, PO Box 124 SE-22100 Lund, Sweden. ${ }^{2}$ CNR-ISC and Department of Physics, Sapienza University of Rome, Piazzale A. Moro 2, 00185 Roma, Italy. ${ }^{3}$ Present address: CR Competence AB, Naturvetarevägen 14, 22362 Lund, Sweden. ${ }^{4}$ Present address: Department of Physics, University of Konstanz, PO Box 688D-78457 Konstanz, Germany. Correspondence and requests for materials should be addressed to E.Z. (email: emanuela.zaccarelli@cnr.it) or to P.S. (email: peter.schurtenberger@fkem1.lu.se)
} 
M icrogels are hybrid particles with a dual colloid-polymer nature, belonging to the class of so-called soft colloids ${ }^{1,2}$. A microgel consists of a mesoscopic crosslinked polymer network, which can deform, shrink or interpenetrate with another microgel ${ }^{3,4}$. Often, as a result of the synthesis conditions, a particle possesses a denser core and a more loosely crosslinked corona ${ }^{5,6}$, which also includes so-called dangling ends ${ }^{7,8}$. Microgels are considered smart colloidal materials: in response to external parameters such as temperature, $\mathrm{pH}$, ionic strength, light, or electric field (depending on the nature of the polymers) a particle is able to change its size as well as other connected properties such as the polarizability ${ }^{9}$ or elasticity of the particle ${ }^{10,11}$. Thus, they are promising for and already employed in several applications, such as photonic crystals ${ }^{12,13}$, drug delivery systems ${ }^{14-16}$, or nanotechnologies ${ }^{17}$. In addition, thanks to their high tunability and to their softness, microgels represent ideal model systems to study phase transitions ${ }^{18-22}$ and glass or jamming transitions in dense colloidal dispersions ${ }^{23-25}$.

In the case of thermoresponsive microgels made of poly $(\mathrm{N}-$ isopropylacrylamide) (PNIPAM), the soft colloids are swollen below the volume phase transition temperature (VPTT) of $32^{\circ}$ $\mathrm{C}^{26-28}$. At temperatures $T>\mathrm{VPTT}$, the swollen microgel network collapses and expels a significant fraction of water ${ }^{27,29}$. Thus, temperature is readily used as a convenient parameter to control in situ the size and the volume fraction of microgel samples ${ }^{18-21,25,30}$. In doing so, however, one implicitly assumes that such a temperature change does not alter the effective interactions between microgels. Early studies have proposed to model effective interactions between swollen microgel particles (below the VPTT) in terms of a hard-sphere-like potential, with a modified/effective hard sphere diameter ${ }^{5,31,32}$, whereas for $T>$ VPTT microgels should behave as attractive spheres ${ }^{32}$. Recent research shows that the interactions between swollen microgels can be more accurately reproduced by a soft Hertzian repulsion in the fluid region of the phase diagram ${ }^{33}$, while brush-like models can be used for highly packed samples ${ }^{34,35}$. All these different models point to the surprising fact that there is not yet a unifying picture which can describe microgels' interactions. Clearly there is the need to carefully characterize the interparticle potential under different experimental conditions across the entire phase space. Such a step is necessary not only for a correct use of microgel systems in their widespread applications but also from a fundamental point of view: only fully characterized systems should be used to work on open problems in condensed matter physics, such as glass transition and jamming.

In this work, we investigate the effective interactions of microgels in a wide region of the fluid regime, and as a function of temperature for $T<$ VPTT, i.e., for swollen microgels. We study both one-component microgel suspensions and binary mixtures in which much smaller microgels are added, inducing an effective depletion on the large ones. For each state point, experimental structural and dynamical information was compared to its simulated counterpart. We confirm the applicability of a soft repulsive Hertzian interaction potential for the onecomponent system, even at elevated temperatures. However, the Hertzian model predicts the instantaneous aggregation of the large microgels in the mixtures, which experience a depletion attraction. In contrast, all mixtures are stabilized by the corerepulsion of the microgels. Based on numerical calculations of the effective interaction potential, we develop a multi-Hertzian (MH) model, which ascribes a different elasticity to corona-corona interactions-reflecting the simple Hertzian interactions between microgels at moderate packing fractionsand core-corona or core-core interactions. The $\mathrm{MH}$ model captures the structure and the dynamical behavior of the studied binary mixtures at all 48 investigated different state points.
Evidently, it is imperative to consider the variation of the interparticle potential upon changing temperature and, crucially, the internal structure of the microgels to correctly describe their behavior, particularly for conditions where microgels are forced together-for example, in electric field applications or in the dense glassy regime, which is most widely studied in the microgel literature. Furthermore, our results raise fundamental questions on the widespread practice to tune the volume fraction via a temperature change without accounting for the different nature of the system.

\section{Results}

Structure and dynamics of one-component microgel systems. We start by analyzing the behavior of one-component microgel suspensions (also referred to as 'colloid' samples) with weight fractions $\mathrm{wt} \%=2.2,3.3,4.4$. The radial distribution function $(g$ $(r))$ and mean squared displacements (MSD) of the samples were measured at four different temperatures in the range $15-30{ }^{\circ} \mathrm{C}$. We find, as expected, that an increase in particle concentration leads to an increase in the structural correlations for all $T$ (Fig. 1a). An increase in temperature is associated with the deswelling of particles, which is quantified by additional dynamic light scattering measurements (Supplementary Fig. 1, Supplementary Note 1), and thus causes a decrease of the volume fraction of the sample. This leads to a reduced structural order as well as to the shift of the main peak of the $g(r)$ toward smaller distances. From the trajectories obtained with confocal laser scanning microscopy (CLSM) we also reconstruct the MSDs in a time window within the long-time diffusive regime of the microgels (Fig. 1b). We find that increasing temperature speeds up particle diffusion, due to the reduction in volume fraction as well as to the faster thermal motion and to a reduction in solvent viscosity.

In order to describe the experimental behavior, we use the soft Hertzian-type repulsion which has been previously shown to accurately describe microgel interactions in the fluid phase at $15^{\circ}$ $\mathrm{C}^{33,36}$. The (colloid-colloid) Hertzian potential $V_{\mathrm{cc}}^{\mathrm{H}}(r)=U_{\mathrm{cc}}(1-$ $\left.r / \sigma_{\text {eff }}\right)^{5 / 2} \theta\left(\sigma_{\text {eff }}-r\right)$ where $\theta(r)$ is the Heaviside step function, depends on two control parameters: the effective colloid diameter $\sigma_{\text {eff }}$ and the interaction strength at full overlap $U_{\text {cc }}$. We fix the former to be equal to $2 R_{\mathrm{H}}$, where $R_{\mathrm{H}}$ is the (experimentally determined) hydrodynamic radius of the particles at each considered $T$ (Supplementary Fig. 1, Supplementary Note 1). Next, we adjust the colloid volume fraction $\phi_{\text {eff,c }}$ at $T=15^{\circ} \mathrm{C}$ around the value predicted by viscometry (see Methods, Supplementary Fig. 2, Supplementary Note 2) and we vary $U_{\mathrm{cc}}$ until a good correspondence is found with the experimental $g(r) s$.

We find, in line with previous work with slightly different microgels $s^{33}$, that the interaction strength at $T=15^{\circ} \mathrm{C}$ is $U_{\mathrm{cc}}=$ $400 k_{\mathrm{B}} T$ and the three colloid packing fractions, that will serve as a basis also for the binary mixtures discussed later on, are $\phi_{\text {eff,c }}=$ $0.26,0.37$ and 0.49 at $15^{\circ} \mathrm{C}$. To model the variation in temperature, the volume fractions are changed according to the deswelling of the microgels (Supplementary Fig. 1, Supplementary Note 1), and again we vary the interaction strength $U_{\mathrm{cc}}$ until the experimental radial distribution functions are well reproduced also at higher $T$. Consequently, we find that the numerical and experimental $g(r)$ are in good agreement for all investigated state points. In particular, the positions of all peaks is well-captured and the secondary peaks are quantitatively reproduced, while some deviations are observed close to the main peak. However, there is no systematic trend of such deviations with respect to packing fraction, as shown and described in Supplementary Fig. 3 and Supplementary Note 3. This suggests that the discrepancy is mostly driven by data noise. A systematic worsening of the 

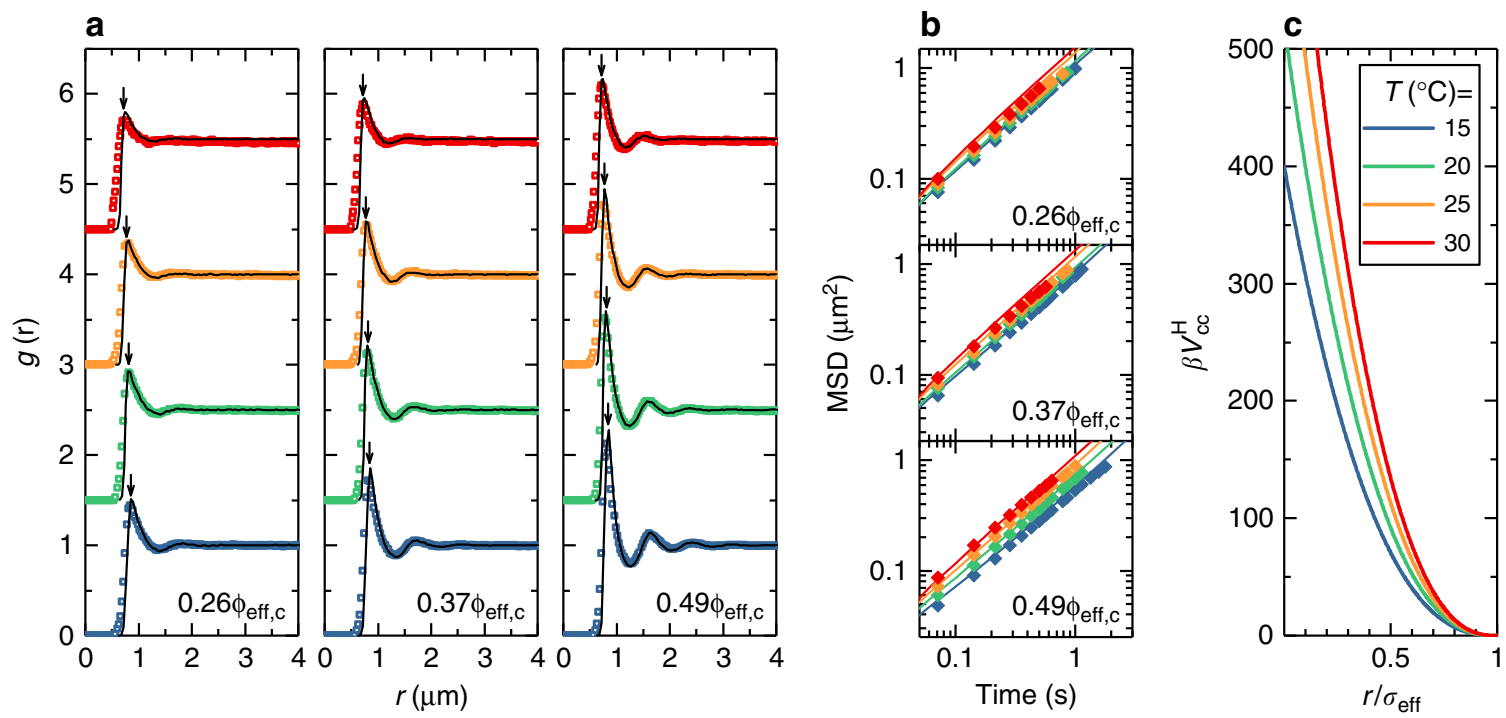

Fig. 1 Temperature-dependent structure, dynamics and interaction potential of one-component microgel suspensions. Symbols indicate experimental data, solid lines represent simulations. Color legend applies to all panels. a Radial distribution functions $g(r)$ from simulations and experiments. Panels show data for $\phi_{\text {eff, }}=0.26,0.37$ and 0.49 (left to right, evaluated at $T=15^{\circ} \mathrm{C}$ ) for $15^{\circ} \mathrm{C} \leq T \leq 30^{\circ} \mathrm{C}$. Graphs are offset along the $y$-axis for clarity. Downward pointing arrows indicate the hydrodynamic diameter of colloids at each $T$. $\mathbf{b}$ MSDs for $15^{\circ} \mathrm{C} \leq T \leq 30^{\circ} \mathrm{C}$ reconstructed from the $x, y$ trajectories, i.e. $\left\langle x^{2}+y^{2}\right\rangle$, with $\phi_{\text {eff, },}=0.26,0.37$ and 0.49 (from top to bottom). c Hertzian interaction potential at different temperatures: $U_{\mathrm{cc}}=400,520,640,760 \mathrm{k}_{\mathrm{B}} T$ for $T=15,20$, $25,30^{\circ} \mathrm{C}$ respectively. The distance is rescaled by $\sigma_{\text {eff }}=2 R_{\mathrm{H}}$

agreement is found at $T=30^{\circ} \mathrm{C}$, where lower spatial resolution of the CLSM in the $z$-direction and the rapid Brownian motion of the particles leads to a reduction in the peak height and a broadening of the $g(r)$ data for values to the left of the first peak ${ }^{33}$.

The agreement between experimental and numerical data indicates that the Hertzian model is able to describe the structure of the swollen microgel system in the range of investigated packing fractions. Furthermore, we find that $U_{\mathrm{cc}}$ approximately follows a linear dependence on temperature (Supplementary Fig. 5, Supplementary Note 5). The fact that raising the temperature makes PNIPAM more hydrophobic might give rise to expectations that by increasing $T$ microgels become more and more attractive 29,32 . By contrast, our results show that the Hertzian repulsion is an increasing rather than decreasing function of temperature, at least up to $30^{\circ} \mathrm{C}$ (Fig. 1c), in agreement with static light scattering experiments previously obtained with microgels with a lower crosslink density ${ }^{32}$. Only close to the VPT and beyond, outside of the regime explored in this work, attractive interactions become dominant ${ }^{32}$.

A further test of the Hertzian model can be made by comparing experimental and numerical MSDs. To this aim we use Brownian Dynamics (BD) simulations, which show that that the Hertzian model is also able to reproduce the variation of the MSDs (Fig. 1b) with $T$ and $\phi_{\text {eff,c }}$ in the investigated regime. The direct comparison between the numerical and experimental selfdiffusion coefficients is reported in Supplementary Fig. 4 and Supplementary Note 4.

It is particularly interesting to directly compare samples with the same effective volume fraction but at different temperatures, i.e. samples with unequal number density, as shown in Fig. 2. Comparing the experimental data for $g(r)$, we find a weak but detectable increase of the correlation with increasing temperature. Indeed, at the higher $T$ the first two peaks increase their height and shift towards larger separations, the in-between minimum deepens and a weak oscillation beyond $r / \sigma_{\text {eff }}=2$ appears. Not only the structure of the system is affected, but also the dynamics. After appropriately rescaling the data to take into account the different hydrodynamic radii and zero-colloid limit diffusion coefficients at each $T$, the experimental MSDs show a marked difference between the two samples: the $T=25^{\circ} \mathrm{C}$ system is much slower than the one at lower temperature. Thus, at higher $T$ the system is more structured, which is consistent with the stronger Hertzian repulsion that we have determined within our theoretical analysis. However, it is important to stress that the present evidence is based solely on experimental data and does not rely on the particular choice of any model. Our measurements clearly show that the interaction potential between the particles changes as a function of temperature, even within the swollen regime $\left(15-30^{\circ} \mathrm{C}\right)$.

To summarize, we have found that a temperature-dependent Hertzian model can be used to correctly capture both the structure and the dynamics of one-component microgel suspensions in the investigated temperature and packing fraction range. Importantly, the Hertzian repulsion is found to increase with temperature. These findings directly confirm the hypothesis that, by changing the temperature, not only the packing fraction is varied but also the interaction potential is considerably affected. This is particularly important for studies in which the temperature is used as a facile way to tune the effective volume fraction of soft microgels, where these temperature-dependent changes in interparticle interactions should be carefully considered.

The Hertzian model poorly describes microgel mixtures. We now turn to analyze mixtures of large (colloid) and small (depletant) microgels. The very small size ratio $R_{\mathrm{H}, \text { depletant }} / R_{\mathrm{H}}$, colloid changes very little, i.e. from 0.055 to 0.060 , within the investigated temperature range (Supplementary Fig. 1, Supplementary Note 1). In this framework, it is possible to derive an effective interaction potential for large microgels only, integrating out the small particles' degrees of freedom ${ }^{37}$. The small microgels thus induce a depletion interaction between the colloids.

We investigate nine colloid-depletant mixtures with colloid wt $\%=2.2,3.3,4.4$ and depletant $\mathrm{wt} \%=0.26,0.54,0.81$. We start by analyzing structural correlations (Fig. 3). In the presence of 

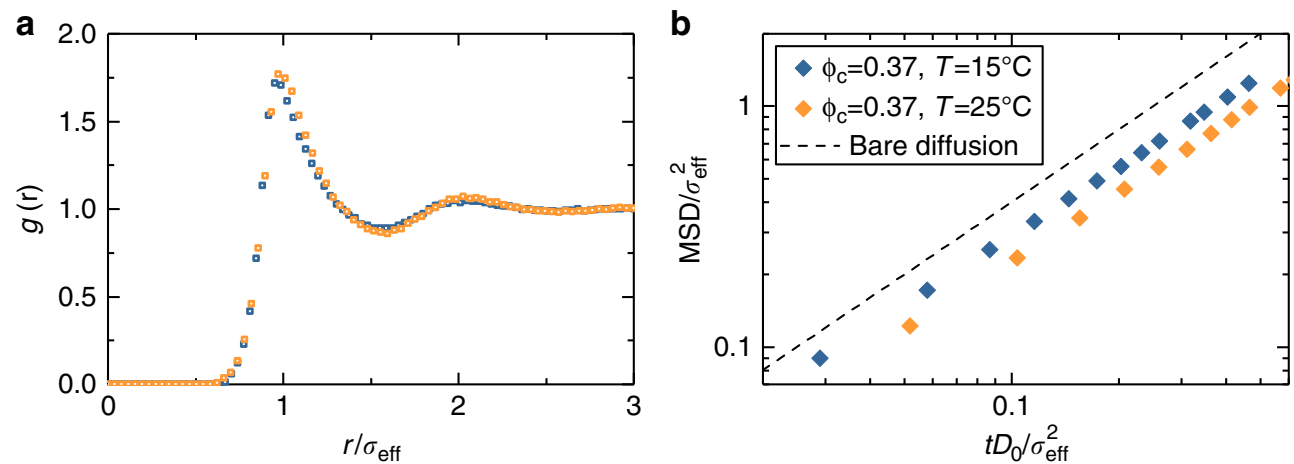

Fig. 2 Structure and dynamics for two state points at different temperature with equivalent packing fraction. (a) Experimental $g(r)$ and (b) MSD for samples with $\phi_{\text {eff,c }}=0.37$ at two different temperatures $\left(T=15\right.$ and $\left.25^{\circ} \mathrm{C}\right)$ corrected for size. $D_{0}$ is the zero-colloid limit diffusion coefficient and its associated MSD (dashed line) is also shown

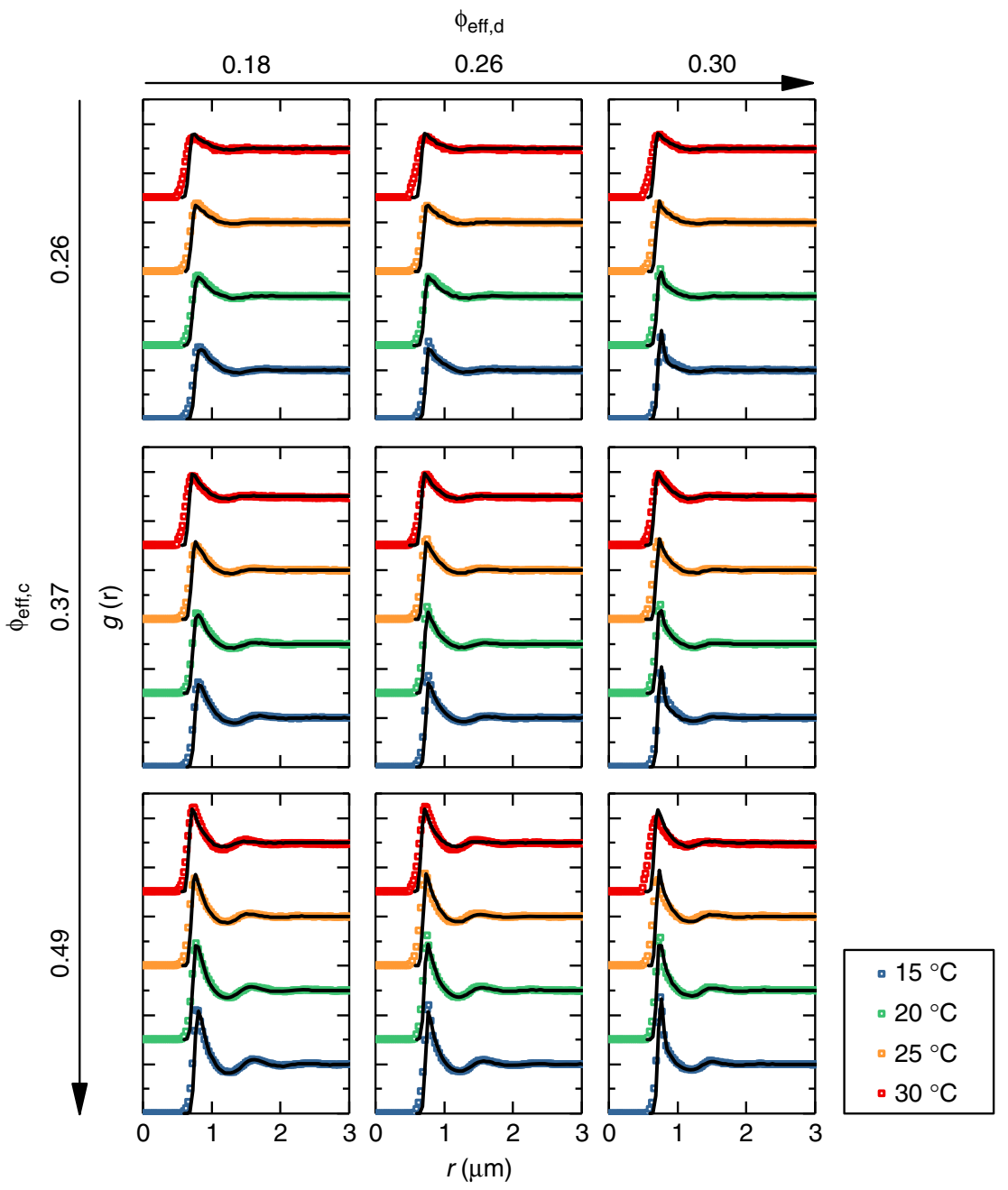

Fig. 3 Experimental and numerical structural correlations for all investigated binary mixtures. Experimental $g(r) s$ (colored squares) are compared to numerical ones (solid lines) based on the multi-Hertzian model. Data for different samples are offset in $y$ for clarity. The color legend applies to the entire graph. Values of $\phi_{\mathrm{eff}, \mathrm{c}}$ and $\phi_{\mathrm{eff,d}}$ at $15^{\circ} \mathrm{C}$ are given for each row and column, respectively. For higher temperatures, the values of $\phi_{\mathrm{eff}, \mathrm{c}}, \phi_{\mathrm{eff}, \mathrm{d}}$ can be found in Table 1

depletants, the colloidal particles show an increased attraction: the first maximum of the $g(r)$ increases in height and becomes asymmetric. In addition, the nearest neighbor distance, characterized by the position of such maximum, decreases with the addition of depletants.
A striking result from the experiments is that the depletion attraction is not as strong as expected: all studied mixtures are surprisingly stable and fluid-like. Comparing with recent results for a binary mixture of hard spheres (colloids) and microgels (depletants), phase separation was observed well within the 
currently investigated range of depletant concentrations ${ }^{38}$. Furthermore, theoretical models for depletion among soft particles would predict a strongly enhanced depletion attraction as compared to that occurring in corresponding hard-sphere systems $^{39}$. On the contrary, our experimental findings show that the effect of the depletion attraction is small. This is confirmed by the variation of the MSDs with added depletants (Fig. 4): upon increasing depletant concentration we only observe a moderate slowing down of the diffusion.

In order to describe the observed behavior, we start by modeling the binary mixtures at $T=15^{\circ} \mathrm{C}$ using again the Hertzian model. Thus, the total interaction in the mixture amounts to $V_{\text {tot }}=V_{\mathrm{cc}}^{\mathrm{H}}+V_{\mathrm{cd}}^{\mathrm{H}}+V_{\mathrm{dd}}^{\mathrm{H}}$, where the three terms are the direct colloid-colloid interaction, the colloid-depletant interaction and the direct depletant-depletant interaction, respectively. For the first term $V_{\mathrm{cc}}^{\mathrm{H}}$, we use the previously established model in the absence of depletants, with interaction strength $U_{\mathrm{cc}}=400 k_{\mathrm{B}} T$. To estimate the depletant-depletant term we rely on additional static light scattering measurements for the small microgels (Supplementary Fig. 6, Supplementary Note 6), which lead us to an estimated Hertzian interaction strength at contact of $U_{\mathrm{dd}} \simeq 100 k_{\mathrm{B}} T$.

Assuming additive interactions in the mixture, the crossinteraction strength between large and small microgels would be $U_{\mathrm{cd}}=250 k_{\mathrm{B}} T$. Since simulations of the full binary system are rather costly at the small investigated size ratios, we proceed by assuming ideal depletant-depletant interactions, which simplifies the theoretical description in terms of an (effective) onecomponent system. This assumption is justified by the small size as well as by the very soft interactions between depletants. The interactions between large microgels can thus be calculated as $V_{\mathrm{eff}, \mathrm{cc}}^{\mathrm{H}}=V_{\mathrm{cc}}^{\mathrm{H}}+V_{\mathrm{depl}}$, where $V_{\mathrm{depl}}$ is the additional depletion term induced by the small microgels which depend only on the crossinteractions $V_{\mathrm{cd}}^{\mathrm{H}}$ and on the depletant volume fraction $\phi_{\mathrm{eff}, \mathrm{d}}$, as explained in the Methods.

The resulting interaction potential $V_{\text {eff.cc }}^{\mathrm{H}}$ is far too attractive (Supplementary Fig. 7a, Supplementary Note 7) even at very low $\phi_{\text {eff,d }}$, independently on the choice of $U_{\text {cd }}$. Indeed, even considering rather low (and strongly non-additive) depletantcolloid interactions, the resulting effective potential would lead to instantaneous aggregation between the colloids. In contrast, all studied binary mixtures are experimentally stable. Thus, the Hertzian repulsion model dramatically fails in capturing the behavior of the particles once we add even the smallest amount of attractive depletion.

The multi-Hertzian model for microgel-microgel interactions. The Hertzian model fails to describe binary mixtures, because its soft repulsion is too weak to counteract the depletion attraction. Indeed, for soft, penetrable particles, we have to consider interactions down to $r \rightarrow 0$, where the depletion attraction can become very large ${ }^{39}$. We thus need to model the repulsion between microgels in a more realistic way, taking into account that the density profiles for individual microgels studied in this work show a core-corona structure ${ }^{5,7,8}$. Hence, the addition of depletion interactions allows us to reveal the 'hidden' effect of the microgel core even without directly probing too dense regimes.

In a recent numerical work, some of us have addressed the question of the validity of the Hertzian model by performing numerical simulations of realistic in silico microgel particles ${ }^{8,40}$. We have shown that the Hertzian predictions only hold up to repulsion strengths of $\approx 6 k_{\mathrm{B}} T$ and to packing fractions of order unity. These results confirm that, for one-component microgels in the range of $\phi$ investigated here, we can successfully describe the system properties with the Hertzian model, with the strength of the repulsion being linked to the elastic moduli of the microgels, which can be computed independently ${ }^{40}$. For smaller separations, when the repulsion between two microgels sensibly exceeds the thermal energy, the interaction acquires a clear non-Hertzian nature, as shown in Fig. 5a. Interestingly, here we find that the full dependence of the effective interaction on the microgel-microgel separation can be fitted to a cascade (three in the example shown in Fig. 5) of Hertzian potentials. The very good quality of the fit can be understood in terms of the microscopic architecture of the microgel, which can be considered to be composed by a sequence of more and more dense shells, each of them corresponding to a different internal elasticity and thus to a different Hertzian contribution. Thus, while the Hertzian model is only able to capture the interactions between the outer parts of the coronas of the two microgels, stronger repulsions need to be considered in order to include core-corona and core-core contributions. Such a

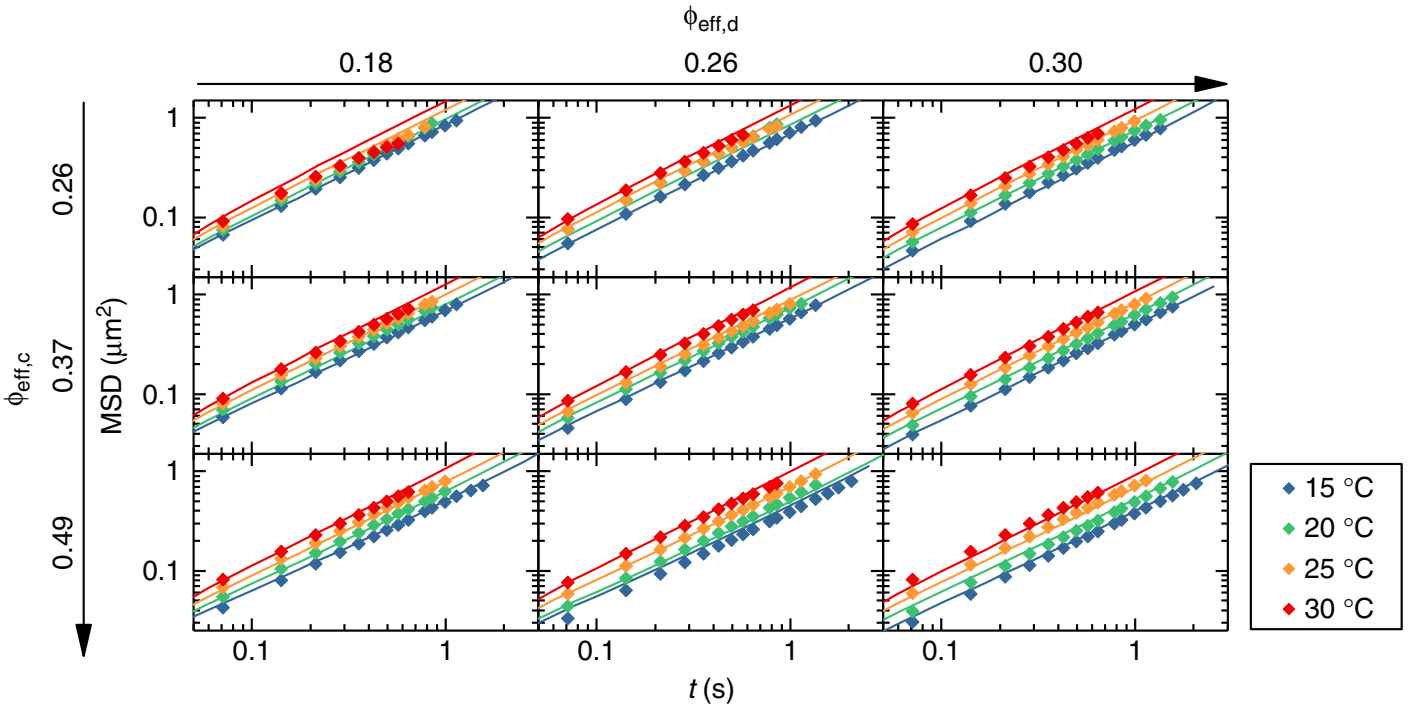

Fig. 4 Experimental and numerical mean square displacements for all investigated state points. Diamonds denote $2 \mathrm{D}$ experimental data $\left(\left\langle x^{2}+y^{2}\right\rangle\right)$, while solid lines represent the corresponding simulation results based on the $\mathrm{MH}$ model. The color legend applies to the entire graph. $\vee$ alues of $\phi_{\text {eff,c }}$ and $\phi_{\text {eff,d }}$ at $15^{\circ} \mathrm{C}$ are given for each row and column, respectively. For higher temperatures, the values of $\phi_{\text {eff,c }} \phi_{\text {eff,d }}$ can be found in Table 1 

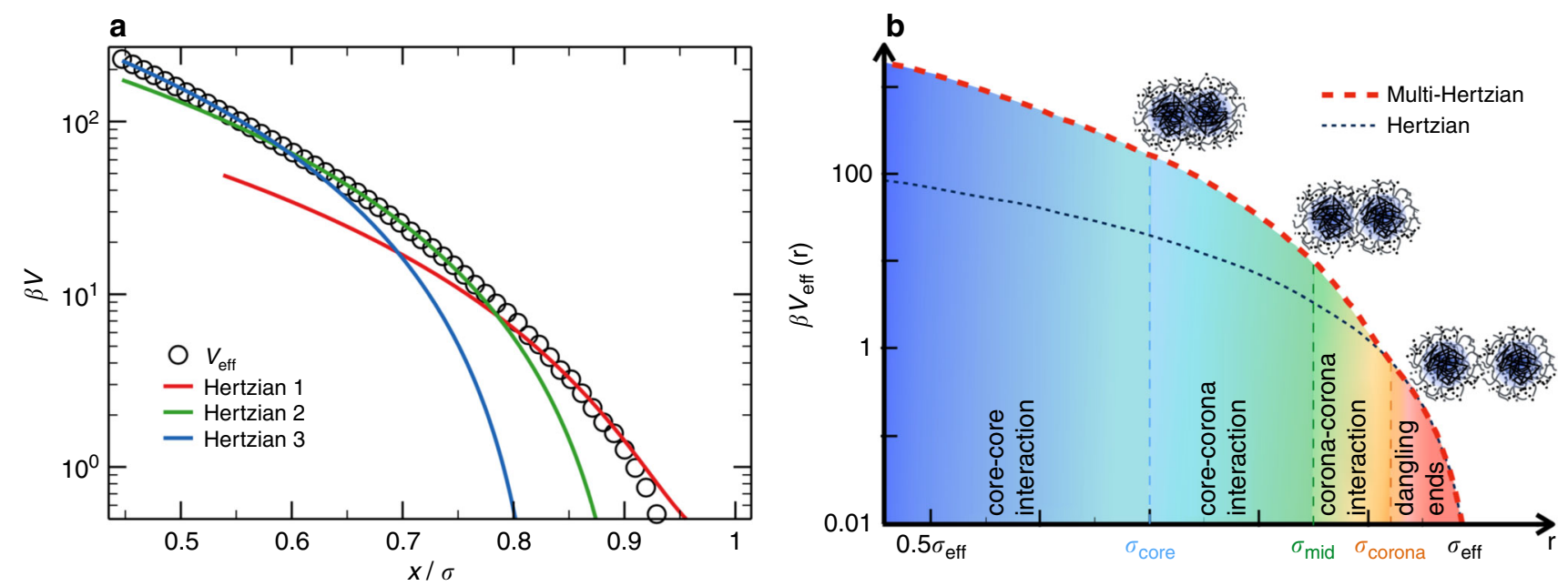

Fig. 5 The multi-Hertzian model. a Calculated effective potential between two microgels as a function of their center-to-center distance. Lines are fits to three different Hertzian contributions, labeled respectively as Hertzian 1, which corresponds to the calculated elastic moduli 40 and whose effective diameter $\sigma$ is used to rescale the $x$-axis, Hertzian 2 and Hertzian 3 representing the contributions of the inner structure of the microgels. For the reported microgel, the fitted strengths are $U_{1}=335 k_{\mathrm{B}} T, U_{2}=1182 k_{\mathrm{B}} T$ and $U_{3}=2617 k_{\mathrm{B}} T$ and the fitted lengths are $\sigma_{1}=\sigma_{,} \sigma_{2}=0.92 \sigma$ and $\sigma_{3}=0.8354 \sigma$ in good qualitative agreement with the ones used to fit experimental data whose parameters are given in Table 2; $\mathbf{b}$ the model describing experimental data with the employed interactions lengths: $\sigma_{\text {core, }}$ below which core-core interactions take place, $\sigma_{\text {mid }}$ relevant to the onset of core-corona interactions and $\sigma_{\text {corona }}$ which reflect the heterogeneous nature of the outer corona shell. A comparison with the Hertzian model is also provided. Note the logarithmic scales on the $y$-axis for both panels

multi-Hertzian model is able to describe the numerically calculated potential up to the smallest simulated particleparticle distances, which correspond to strengths of order $200 k_{\mathrm{B}} T$ for the considered microgel.

We thus apply the $\mathrm{MH}$ model (schematically illustrated in Fig. 5) to the investigated binary mixtures, fixing most of the model parameters according to experimental data as described in Methods. It turns out that we need to take into account four successive shells, reading as

$$
\begin{aligned}
V_{\mathrm{cc}}^{\mathrm{MH}}(r)= & U_{\mathrm{cc}}\left(1-r / \sigma_{\text {eff }}\right)^{5 / 2} \theta\left(\sigma_{\text {eff }}-r\right)+U_{\text {corona }}\left(1-r / \sigma_{\text {corona }}\right)^{5 / 2} \theta\left(\sigma_{\text {corona }}-r\right) \\
& +U_{\text {mid }}\left(1-r / \sigma_{\text {mid }}\right)^{5 / 2} \theta\left(\sigma_{\text {mid }}-r\right)+U_{\text {core }}\left(1-r / \sigma_{\text {core }}\right)^{5 / 2} \theta\left(\sigma_{\text {core }}-r\right)
\end{aligned}
$$

where the outermost shell, extending up to $\sigma_{\text {eff, }}$ coincides with the Hertzian model of strength $U_{\mathrm{cc}}$. This ensures that, in the investigated regime, the behavior of the one-component microgel system is the same for the MH model and for the Hertzian model (Supplementary Fig. 8, Supplementary Note 8). The size of the innermost shell is set by $\sigma_{\text {core }}$, which is the experimentally determined core size and indicates the onset of core-core interactions with a very large repulsion strength $U_{\text {core }}$. Because the transition from the core to the corona is gradual, we introduce an intermediate shell at the midpoint $\sigma_{\text {mid }}$, signaling core-corona interactions. We find that the introduction of an additional elasticity within the outer shell (starting at $\sigma_{\text {corona }}$, the midpoint of the corona) is necessary to reproduce the experimental data to differentiate the contribution of the dangling ends ${ }^{7}$ of the order of $\sim k_{\mathrm{B}} T$ from the corona one. This turns out to be slightly different from the numerical result in Fig. 5a, probably due to the small size of the investigated microgels in simulations and to the absence of true dangling ends in this representation. At each of the characteristic lengths of the potential (see Fig. 5b), an associated interaction strength is estimated by simple arguments (see Methods), except for $U_{\text {corona, }}$ which is adjusted to match the experimental data. The obtained strengths are in qualitative agreement with those resulting from the $\mathrm{MH}$ fit of the calculated effective potential.

Developing the multi-Hertzian model at $15^{\circ} \mathrm{C}$. We apply the $\mathrm{MH}$ model to binary mixtures of large ('colloid') and small ('depletant') microgels. The resulting effective potential is now the sum of the multi-Hertzian model for the direct colloid-colloid interactions and the depletion term, as $V_{\mathrm{eff}, \mathrm{cc}}^{\mathrm{MH}}=V_{\mathrm{cc}}^{\mathrm{MH}}+V_{\mathrm{depl}}$. The latter term contains the cross-interactions between the two types of microgels (colloid-depletant interactions), which for consistency should also take a multi-Hertzian form. However, we have explicitly checked that its inclusion makes no significant difference to using a simple Hertzian. Rather, it complicates the description, so that we stick to the simple Hertzian $V_{c d}^{\mathrm{H}}$, whose strength $U_{\text {cd }}$ has yet to be determined. Also, we need to determine the effective depletant volume fraction $\phi_{\text {eff,d }}$.

We start by considering $T=15^{\circ} \mathrm{C}$ and simultaneously vary the free parameters $U_{\text {corona }}, U_{\text {cd }}$ and $\phi_{\text {eff,d }}$ until we find an optimal agreement to reproduce the measured $g(r)$. The resulting effective potential which best describes the experimental data is found for $U_{\text {corona }}=8.25 \times U_{\text {cc }}=3300 k_{\mathrm{B}} T$ and $U_{\text {cd }}=80 k_{\mathrm{B}} T$ (Supplementary Fig. 7b, Supplementary Note 7). Effective depletant volume fractions are $\phi_{\text {eff,d }}=0.18,0.26,0.30$ at $15^{\circ} \mathrm{C}$. The calculated $g(r) \mathrm{s}$ are shown as lines in Fig. 3 and are found to reproduce the behavior of the measured data for all depletant and colloid volume fractions at the examined temperature. Particularly noteworthy is the development of an asymmetric main peak of $g(r)$ at the highest studied volume fractions, which is accurately captured by the MH model.

These findings point out the strongly non-additive character of the interactions in microgel mixtures: indeed the colloiddepletant $U_{\mathrm{cd}}$ is significantly lower than the average of the two individual interactions for colloid and depletant microgels. This is probably due to the ability of soft particles to deform or overlap with each other, differently from hard particles. A possible explanation is that the very small depletant here involved can quite freely interpenetrate within the corona of the large ones, 
modifying the cross-interactions. The non-additivity is thus the key ingredient which allows us to explain the surprising stability of our soft binary mixtures ${ }^{41,42}$. Indeed, thanks to this feature, particles are able to experience a much more moderate depletion attraction than what is observed in hard colloid-soft depletant mixtures ${ }^{38}$ and in additive soft ones ${ }^{39}$. Hence our soft mixtures will eventually phase separate only at much larger depletant concentrations.

Using the multi-Hertzian model at higher temperatures. The incorporation of the temperature dependence is a first real test to the robustness of the MH model. With increasing temperature, the interactions between the colloids in the binary mixtures change. Increasing the temperature has an effect not only on all interactions in the MH model, but also on the colloid-depletant cross interaction $U_{\mathrm{cd}}$ and on the effective volume fractions $\phi_{\mathrm{eff}, \mathrm{c}}$ and $\phi_{\text {eff,d. }}$ The two-volume fractions are easily dealt with: the deswelling of the microgels (in both cases) automatically yields the volume fractions at higher temperatures (see Table 1). For the MH model parameter estimate, we use the temperature dependence of the Hertzian term (see also Fig. 1b) for the outermost corona. We further note that the core size is temperature independent, based on previously published experimental data ${ }^{33}$ and our own unpublished work. Thus, the intermediate shells in the $\mathrm{MH}$ model become thinner and their associated strengths are chosen as done for $15^{\circ} \mathrm{C}$. A detailed description of the choice of parameters is given in the Methods section, but it is important to stress that the temperature dependence of the $\mathrm{MH}$ model has zero free parameters: everything is fixed based on experimental data and the parameters found for $15^{\circ} \mathrm{C}$. Thus the only parameter left to vary is the colloid-depletant cross interaction $U_{c d}$. Once a good agreement with experimental data is found, it is checked a posteriori that the estimated values are very reasonable and obey a roughly linear relation to temperature, analogously to $U_{\mathrm{cc}}$ (Supplementary Fig. 5, Supplementary Note 5).

\section{Table 1 Summary of effective volume fractions for all samples at all temperatures}

\begin{tabular}{lllll}
\multirow{2}{*}{$\left({ }^{\circ} \mathbf{C}\right)$} & one-component system & \multicolumn{3}{c}{ binary mixtures } \\
\cline { 3 - 5 } & $\boldsymbol{\phi}_{\text {eff,c }}$ & $\boldsymbol{\phi}_{\text {eff,d }}$ & $\boldsymbol{\phi}_{\text {eff,d }}$ & $\boldsymbol{\phi}_{\text {eff, }}$ \\
\hline 15 & 0.26 & 0.18 & 0.26 & 0.30 \\
15 & 0.37 & 0.18 & 0.26 & 0.30 \\
15 & 0.49 & 0.18 & 0.26 & 0.30 \\
20 & 0.22 & 0.17 & 0.24 & 0.28 \\
20 & 0.315 & 0.17 & 0.24 & 0.28 \\
20 & 0.42 & 0.17 & 0.24 & 0.28 \\
25 & 0.19 & 0.16 & 0.23 & 0.26 \\
25 & 0.275 & 0.16 & 0.23 & 0.26 \\
25 & 0.37 & 0.16 & 0.23 & 0.26 \\
30 & 0.155 & 0.145 & 0.215 & 0.24 \\
30 & 0.22 & 0.145 & 0.215 & 0.24 \\
30 & 0.29 & 0.145 & 0.215 & 0.24 \\
\end{tabular}

The experimental $g(r)$ s for the binary mixtures are compared with the simulated data in Fig. 3 for all investigated $T$. The final model parameters are reported in Table 2. We find that the $\mathrm{MH}$ model captures all the distinct features of the depletion attraction: the peak shift, its increase and asymmetry all emerge with increasing $\phi_{\text {eff,d }}$ (Fig. 3). It is worth to stress that the agreement of the model with experiments spans 48 different state points and is based essentially on adjusting two parameters: the strength of the second corona shell $U_{\text {corona }}$ (only determined at $15^{\circ} \mathrm{C}$ ) for the $\mathrm{MH}$ model and the cross-interaction strength $U_{\mathrm{cd}}$ (adjusted at each temperature) for the depletion interaction. Thus the present findings represent a strong test in favor of the validity of the present model.

In order to better visualize the effect of temperature, Fig. 6 shows the results for the state point with the largest colloid and depletant volume fractions $\left(\phi_{\text {eff,c }}=0.49\right.$ and $\phi_{\text {eff, }}=0.30$ at $15^{\circ}$ C). An increase of $T$ again reduces the structural correlations and also the effect of depletion (due to the smaller effective depletant volume fraction), which manifests itself at each temperature by an increased asymmetry and by a shift of the main peak of the $g(r)$ toward smaller values of $r$ compared to the one-component system. The peak position is found at smaller distances with respect to the hydrodynamic radius of the colloids, clearly indicating that the particles partially overlap. The agreement of the $\mathrm{MH}$ model with experiments becomes worse for $30^{\circ} \mathrm{C}$, similarly to the case of the one-component system and probably due to the larger statistical noise in the experimental values. The average deviations between numerical and experimental curves are reported in Supplementary Fig. 3 and Supplementary Note 3.

A second robustness test for the $\mathrm{MH}$ model is carried out by calculating the MSD and comparing it with experiments. Similarly to what has been done for the one-component systems, we rely on $\mathrm{BD}$ simulations and compare the calculated and measured MSD of large microgels for each of the nine mixtures in the temperature range $15 \mathrm{C} \leq T \leq 30^{\circ} \mathrm{C}$. As shown in Fig. 4, the current model is also able to capture the particle dynamics for all studied state points. This is confirmed in Supplementary Fig. 4 and Supplementary Note 4, where the self-diffusion coefficients for all state points are shown and described. The small deviations between experiments and simulations observed at $30^{\circ} \mathrm{C}$ can again be rationalized by the larger tracking errors associated to the rapid Brownian motion of the microgels at this temperature.

\section{Discussion}

In this study, we have presented an extended investigation of microgel suspensions in a three-axis phase diagram. In addition to varying microgel volume fraction and temperature, we also varied the concentration of a second component in the suspension, namely smaller microgels, which act as depletants. We investigate one-component and binary mixtures of microgels in a wide range of control parameters, amounting to 48 different state points. Through the combination of confocal microscopy experiments and simulations, we provide a systematic and comprehensive characterization of both static and dynamic observables in the

Table 2 Summary of all parameters for the MH model and depletion term as a function of temperature

\begin{tabular}{lllllllll}
$\boldsymbol{T}\left({ }^{\circ} \mathbf{C}\right)$ & $\boldsymbol{U}_{\text {core }}$ & $\boldsymbol{U}_{\text {mid }}$ & $\boldsymbol{U}_{\text {corona }}$ & $\boldsymbol{U}_{\mathbf{c c}}$ & $\boldsymbol{U}_{\text {cd }}$ & $\boldsymbol{\sigma}_{\text {core }}$ & $\boldsymbol{\sigma}_{\text {mid }}$ & $\boldsymbol{\sigma}_{\text {corona }}$ \\
\hline 15 & 10000 & 4000 & 3300 & 400 & 80 & 0.70 & 0.85 & 0.925 \\
20 & 10000 & 5200 & 4300 & 520 & 108 & 0.74 & 0.87 & 0.935 \\
25 & 10000 & 6400 & 5300 & 640 & 144 & 0.77 & 0.885 & 0.945 \\
30 & 10000 & 7600 & 6300 & 760 & 180 & 0.83 & 0.915 & 0.960 \\
\hline
\end{tabular}

Hertzian strengths $U_{\text {core, }} U_{\text {mid, }} U_{\text {corona }}$ and $U_{c c}$ in $k_{\mathrm{B}} T$ and lengths $\sigma_{\text {core, }} \sigma_{\text {mid }} \sigma_{\text {corona }}$ and $\sigma_{\text {eff }}$ (in units of $\sigma_{\text {eff }}$ ) as used for colloid-colloid interactions. Hertzian strength $U_{c d}$ in $k_{\mathrm{B}} T$ used to describe the colloid-depletant (non-additive) interaction in binary mixtures 

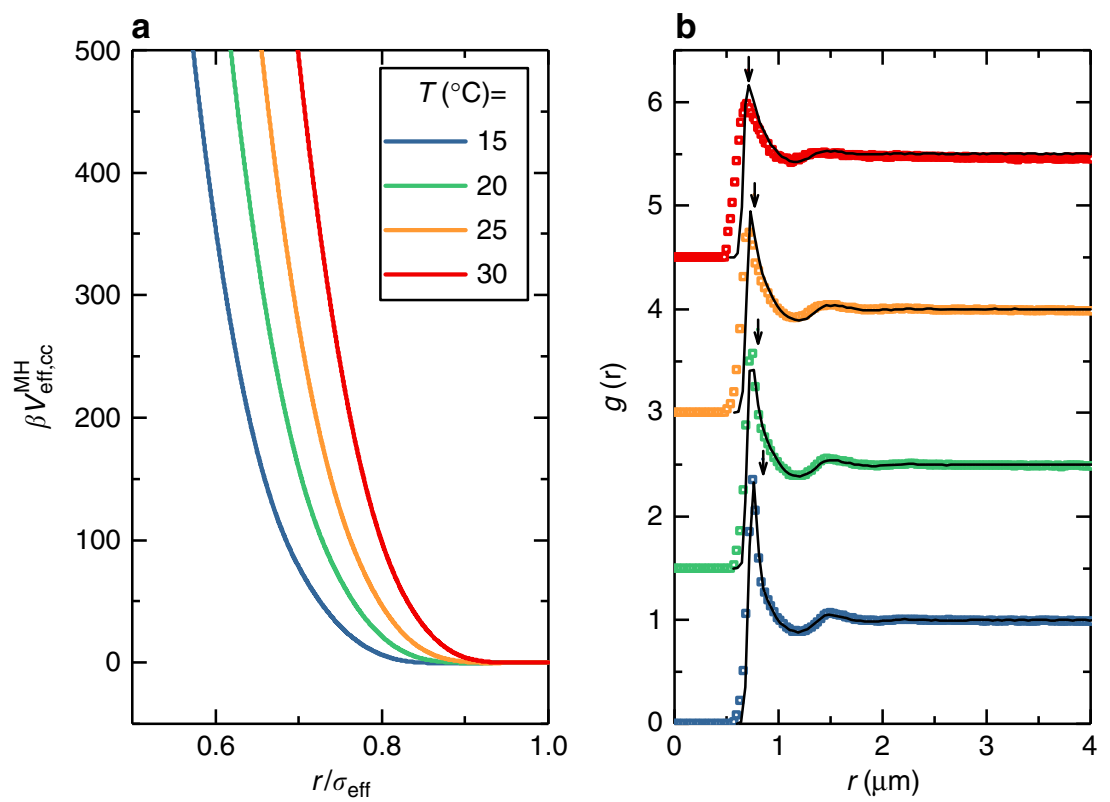

Fig. 6 Typical $V_{\text {eff,cc }}^{\mathrm{MH}}$ and resultant $g(r)$ s for one state point at different temperatures. a Calculated effective potential $\beta V_{\text {eff,cc }}^{\mathrm{MH}}$ based on temperaturedependent $V_{c c}^{\mathrm{MH}}$ and $V_{\mathrm{cd}}$. At $T=15^{\circ} \mathrm{C}, \beta V_{\mathrm{eff}, \mathrm{cc}}^{\mathrm{MH}}$ displays a shallow negative minimum at $\sim-0.6 k_{\mathrm{B}} T$. (b) Comparison between numerical $g(r) \mathrm{s}$ (solid lines) and experimental $g(r) s$ (colored squares). From top to bottom temperature increases from 15 to $30^{\circ} \mathrm{C}$. Downward pointing arrows indicate the hydrodynamic diameter of colloids which does not generally coincide with the first peak position at any temperature

form of radial distribution functions and mean-squared displacements of the large microgels. Based on explicit calculations of the effective potential between two microgels, we have been able to develop a new interaction potential that, with a single set of experiment-informed parameters, is able to reproduce the statics and dynamics of real microgel suspensions, accounting for the dependence on microgel volume fraction, temperature and depletant concentration. Although microgels are nowadays a widely studied model system, such an extensive study was crucially missing. The several novel findings reported here will change the approach to the use of microgels as model systems in future work. Indeed, these soft particles appear to be much more complex systems than naively thought.

First of all, we have provided evidence that the effect of temperature on microgel-microgel effective interactions is not negligible, even within the swollen regime only. The soft Hertzian repulsion between the particles becomes steeper with increasing $T$. This seemingly straightforward result is not obvious since, for $T>$ VPTT, microgels become attractive due to the increased van der Waals and additional hydrophobic interactions. Therefore, an increase of repulsion goes in the opposite direction. The trend can be rationalized by thinking of the microgels only in physical terms (ignoring polymer-solvent interactions which are not yet dominant): as the particles become smaller, they also become more compact and hence somewhat less penetrable. A further change in interactions at high $T$ is however hinted by the present results, as the simple repulsive model that we have adopted shows increasing deviations at the highest studied $T=30^{\circ} \mathrm{C}$, approaching the VPTT at $32{ }^{\circ} \mathrm{C}$. Close to the VPTT, a much more careful evaluation, also in terms of charge effects which could become important as shown by our and others preliminary measurements ${ }^{43}$, will be required. For the examined $T$-interval, the present findings clearly show that the variation of volume fraction that is obtained by changing $T$, a commonly used method in experiments to efficiently explore a larger portion of the phase diagram, should be done with caution, as doing so significantly affects the effective interactions between the particles. Previous works have already pointed out this important aspect through indirect observations ${ }^{29}$, but here for the first time we provide a direct evidence and quantify the change of behavior with $T$ across the swollen regime.

Secondly, we have shown that a simple structureless model such as the Hertzian repulsion does not work to describe conditions where overlaps between particles and/or deformations start to be probed. These effects are an important physical ingredient that deeply affect the behavior of soft colloids in general and of microgels in particular, at the heart of a large research activity on glass transition and jamming of soft particles. Even without directly exploring dense conditions, the use of depletants has allowed us to probe the effective interactions between microgels at short separation distances, finding evidence of the importance of the internal microgel architecture. We have thus transferred our previous knowledge from a simple Hertzian model to a multi-Hertzian one, which is confirmed by explicit calculations of the effective potential between two microgels, that involves the inclusion of inner shells of different elasticity. Interestingly, we find that to successfully describe the experimental data it is important not only to differentiate between core and corona, but also to take into account the heterogenous character of the corona, further differentiating the contribution of the dangling ends ${ }^{7,8}$. Given the numerous studies where different synthesis protocols have been implemented to obtain other internal structures and crosslink density distributions, see e.g. refs $44-47$, it will be interesting to systematically study and quantify how the crosslink density and internal structure of the microgels influence their effective interactions in future studies.

The multi-Hertzian model that we have designed is based on numerical evidence and on available experimental parameters. The comparison with experimental data has allowed us to determine the unknown parameters, most importantly the crossinteractions between small and large microgels. This is a key player in the depletion interaction, and the very low strength that we have determined for cross interactions does explain the striking finding that soft microgel mixtures are much more stable, 
up to very high depletant concentrations, than expected. Indeed, previous works with additive soft mixtures have shown how softness enhances depletion attraction ${ }^{39}$. Here we show that this does not happen, because softness allows deformation and interpenetration, which translates to strongly non-additive interactions. It will be interesting to confirm these findings also for other soft mixtures and in particular, for the more studied classical case of soft colloids and non-adsorbing polymers acting as depletants.

Finally, our phenomenological approach will have to be generalized to deal with different conditions such as even higher $T$ or larger microgel volume fractions, approaching the glass transition. However, the reported evidence clearly shows that future studies will have to explicitly take into account temperature dependence and internal microgel structure to meaningfully describe microgel behavior and to use them as model systems for exploring phase transitions and glassy dynamics.

\section{Methods}

Synthesis. PNIPAM particles were synthesized via precipitation

polymerization $^{27,28}$. NIPAM was re-crystallized in hexane and all other chemicals were used as received. For the large fluorescent microgels (referred to as colloids), $2.004 \mathrm{~g}$ N-isopropylacrylamide (NIPAM, Acros Organics) was dissolved in $82.83 \mathrm{~g}$ of water. $0.136 \mathrm{~g}$ ( $4.98 \mathrm{~mol} \%$ with respect to NIPAM) of the cross-linker N,Nmethylenebis(acrylamide) (BIS, Sigma-Aldrich) was added. $0.002 \mathrm{~g}$ methacryloxyethyl thiocarbonyl Rhodamine B dissolved in $10 \mathrm{~g}$ of water was added to the reaction mixture to covalently incorporate fluorescent sites. The reaction mixture was heated to $80^{\circ} \mathrm{C}$ and bubbled with nitrogen for $30 \mathrm{~min}$. The reaction was then kept under a nitrogen atmosphere. To start the reaction, $0.1 \mathrm{~g}$ KPS in $5 \mathrm{~g}$ water was injected to the mixture. The reaction was then left for $4 \mathrm{~h}$ before the heat was turned off and the solution was left to cool down under constant stirring.

For the small non-fluorescent particle synthesis (referred to as depletants), we followed the same procedure. We combined $1.471 \mathrm{~g}$ of NIPAM, $0.0647 \mathrm{~g}(3.2 \mathrm{~mol} \%$ with respect to NIPAM) of BIS in $96.29 \mathrm{~g}$ water. $0.1929 \mathrm{~g}$ of sodium dodecyl sulphate (SDS, Duchefa Biochemie) was also added to induce the formation of particles with smaller radii. The mixture was heated to $70^{\circ} \mathrm{C}$, bubbled with nitrogen and $0.0539 \mathrm{~g}$ of KPS in $2.0145 \mathrm{~g}$ of water was added to start the reaction. The reaction was then left for $6 \mathrm{~h}$ under a nitrogen atmosphere.

The particle suspensions were cleaned by three centrifugation and re-dispersion series before the suspensions were freeze-dried to remove all water.

Sample preparation. All samples were prepared using the freeze-dried microgels and deionised water (purified with a MilliQ system), as this allows us to control the weight concentration. In order to ensure homogeneous dispersions, samples were thoroughly mixed by vortexing and sonication followed by placing the dispersion on a tumbler for two weeks prior to any experiment.

Using this approach, samples with a wt\%-range from 0.1 to $1 \mathrm{wt} \%$ of colloids and samples with wt\%-range from 0.1 to $0.8 \mathrm{wt} \%$ of depletants were prepared for viscometry experiments. Very dilute colloid and very dilute depletant suspensions $(<0.1 \mathrm{wt} \%)$ were made for DLS characterization. Suspensions were diluted until almost completely transparent to avoid multiple scattering. For the SLS measurements, samples with a wt\%-range from 0.05 to $0.65 \mathrm{wt} \%$ depletant were prepared. For the CLSM experiments, we aimed for binary mixtures with effective colloid volume fraction $\phi_{\text {eff,c }}=0.2,0.3,0.4$, and with additional effective depletant volume fraction $\phi_{\text {eff, }}=0,0.1,0.2,0.3$ at $15^{\circ} \mathrm{C}$. As an initial guess for the packing fraction of the samples, we used the shift factor $k=\phi /$ wt $\%$ as determined from viscometry measurements on colloid-only and depletant-only samples (see below for the experimental $k$-values). The binary mixtures contained colloid wt $\% 2.2,3.3$ and 4.4 and depletant wt $\% 0,0.26,0.54,0.81$. Final $\phi_{\text {eff }}$ were determined by fitting $g$ $(r)$ curves, as discussed in the manuscript.

Experiments. The viscosity of colloid-only and depletant-only samples with known wt\%-concentration was recorded using an Ubbelohde viscometer at 15 and $30{ }^{\circ} \mathrm{C}$. Flow times were measured 5-6 times, averaged and divided by the flow time of a water sample to extract the relative viscosity of the samples. The relative viscosity was fitted to the well-known Batchelor equation which holds for colloids in the dilute regime ${ }^{48}: \eta_{\text {rel }}=1+2.5 \phi_{\text {eff }}+5.9 \phi_{\text {eff }}^{2}$ with $\phi_{\text {eff }}=k \times w t \%$. From these fits the shift factor $k$ was determined for 15 and $30^{\circ} \mathrm{C}$. For $20,25^{\circ} \mathrm{C}$, the data was interpolated. $k_{\text {colloid }}=0.091,0.0758,0.061,0.046 \mathrm{wt} \%^{-1}, k_{\text {depletant }}=0.332,0.292$, $0.253,0.214 \mathrm{wt}^{-1}$ for $15-30^{\circ} \mathrm{C}$ respectively. The shift factor was used in sample preparation to estimate $\phi_{\text {eff }}$

Microgels were characterized using dynamic light scattering (DLS) with a goniometer-based light scattering instrument that employs pseudo 2D-cross correlation (3D DLS Spectrometer, LS instruments, Switzerland) with laser wavelength $\lambda=660 \mathrm{~nm}$. DLS measurements were performed over a range of 15-30
${ }^{\circ} \mathrm{C}$ resulting in a swelling curve for both colloids and depletants. The hydrodynamic radii were extracted using a first order cumulant analysis averaged over an angular range of $60-100^{\circ}$, and measured every $10^{\circ}$. To probe the interactions between depletants, static light scattering experiments were performed at several packing fractions, and the small wavevector limit $S(0)$ of the static structure factor $S(q)$ for the small microgels was also obtained.

The binary mixtures were imaged in a Leica SP5 confocal microscope at a frame rate of $13.9 \mathrm{~Hz}$ in the range of $15-30^{\circ} \mathrm{C}$. An excitation wavelength of $543 \mathrm{~nm}$ was used in combination with an oil immersion objective at $\times 100$ magnification and numerical aperture 1.4. The confocal microscope is housed in a temperature regulated box which provides a temperature control with a stability of $\pm 0.2^{\circ} \mathrm{C}$ over the range of temperatures used. Because scanning in the $z$-direction would have been too slow, we made $x y$-videos. Such videos of $512 \times 512 \times 4000$ frames were obtained for at least five different positions in the sample to minimize the effects of local density fluctuations. Videos were taken at $\gtrsim 5$ particle diameters away from the glass to avoid wall influences. The accuracy of the coordinates is estimated to be $\Delta x \approx \Delta y \approx 11 \mathrm{~nm}^{33}$. Using standardized image analysis and particle tracking routines ${ }^{49}$, the $2 \mathrm{D} g(r)$ s and $2 \mathrm{D}$ mean square displacements (MSDs) $\left(\left\langle x^{2}+y^{2}\right\rangle\right)$ were obtained. To ensure the $2 \mathrm{D} g(r)$ corresponds to the $3 \mathrm{D} g(r)$, the approach as described in Mohanty et al. was employed ${ }^{33}$. In brief, a thinner 'slice' of data is created by rejecting out of focus particles, i.e. we only take particles with $z=0$. Even so, there will always remain some variation in the $z$-position of the tracked particles. This has been taken into account in the numerical calculations by adding a suitable noise along one of the axes.

Model and theory. We consider two systems: one-component microgel systems and binary mixtures. Colloids experience a direct colloid-colloid interaction that we model as Hertzian or multi-Hertzian $(\mathrm{MH})$ as described in the manuscript. The presence of depletants leads to an additional attractive interaction between the colloids. Thus, the effective colloid-colloid interaction potential $V_{\text {eff,cc }}$ is the sum of two contributions: $V_{\mathrm{cc}}$ (direct colloid-colloid interaction) and $V_{\text {depl }}$ (depletion interaction), i.e. $V_{\text {eff,cc }}(r)=V_{\text {cc }}(r)+V_{\text {depl }}(r)$. We assume that depletants are ideal Under this assumption, the Fourier components of the additional depletion term can be calculated for any colloid-depletant interaction $V_{\mathrm{cd}}$ as in ref. ${ }^{50}$

$$
-\beta \tilde{V}_{\mathrm{depl}}(k)=\rho_{d}\left[\int \mathrm{d} \mathbf{r}\left(e^{-\beta V_{\mathrm{cd}}(\mathrm{r})}-1\right) e^{\mathrm{ik} \cdot \mathbf{r}}\right]^{2}
$$

Here $\rho_{\mathrm{d}}$ is the reservoir depletant number density and $\beta=1 /\left(k_{\mathrm{B}} T\right)$. In our study, we consider $V_{c d}(r)$ to be a Hertzian potential. After Fourier transforming Eq. (2) we obtain $\beta V_{\text {depl }}(r)$ which is added to $\beta V_{c c}$ at each considered temperature for the binary mixtures to obtain the total interaction potential $\beta V_{\text {eff,cc. }}$. We further check that the use of a multi-Hertzian model for $V_{\mathrm{cd}}(r)$ does not yield a noticeable change on the obtained results.

The $\mathrm{MH}$ model is built up as follows. The outer shell corresponds to the Hertzian soft repulsion: $U_{c c}=400,520,640,760 k_{\mathrm{B}} T$ for $15,20,25,30^{\circ} \mathrm{C}$ which sets in at $r=\sigma_{\text {eff }}$, where $\sigma_{\text {eff }}=2 R_{\mathrm{H}}$ is of course temperature dependent (see also Supplementary Fig. 1 and Supplementary Note 1). The inner shell corresponds to the core and is temperature independent. We estimate the core diameter as $\sigma_{\text {core }}=$ $0.7 \sigma_{\text {eff }}$ thanks to available experimental SAXS data (and related fuzzy-sphere model fits) for similar microgels ${ }^{33}$. We fix $U_{\text {core }}=10^{4} k_{\mathrm{B}} T$, a value compatible with elasticity arguments ${ }^{1}$ which takes into account the high crosslink density in the core. In this way, the innermost and outermost Hertzian terms are completely specified based on experimental data. Since the border between the dense core and loosely crosslinked corona is not so well-defined, we introduce an intermediate point at the midpoint between these two lengths, i.e. $\sigma_{\text {mid }}=0.5\left(\sigma_{\text {core }}+\sigma_{\text {eff }}\right)=$ $0.85 \sigma_{\text {eff. }}$ The associated repulsion strength is arbitrarily chosen to be $U_{\text {mid }}=10 \times$ $U_{\text {cc }}$. We find that it is crucial to describe the corona by using two distinct shells, introducing a second intermediate point $\sigma_{\text {corona }}=0.5\left(\sigma_{\text {mid }}+\sigma_{\text {eff }}\right)=0.925 \sigma_{\text {eff }}$ with its associated strength $U_{\text {corona. }}$. The latter is determined at $15^{\circ} \mathrm{C}$ to be $8.25 \times U_{\text {cc }}$ by comparing simulation results with the experimental $g(r)$ s. This relation and that for $U_{\text {mid }}=10 \times U_{c c}$ are then kept throughout the temperature range.

The effective potential $V_{\text {eff,cc }}$ is then calculated at several depletant volume fractions starting with our initial guess from viscometry. Because of the uncertainty in the experimental packing fraction, we adjust $\phi_{\text {eff,d }}$ in the simulations at $15^{\circ} \mathrm{C}$ until we find good agreement with the experimental data. As described in the manuscript, $\phi_{\text {eff,c }}$ was adjusted in the Hertzian simulations at $15^{\circ} \mathrm{C}$. The thus obtained parameter values for the volume fractions $\left(\phi_{\text {eff,c }}, \phi_{\text {eff,d }}\right)$ and the parameters for the MH model are summarized in Tables 1 and 2, respectively.

To justify the assumption of ideal behavior of small microgels and the nonadditive character of interactions in the mixture, we have further quantified the interactions between small microgels assuming a Hertzian repulsion. To calibrate its strength, we have computed $S(0)$ by solving the Ornstein-Zernike equation within the Rogers-Young closure, finding that a very soft interaction between depletants, i.e. $U_{\mathrm{dd}} \simeq 100 k_{\mathrm{B}} T$, captures the small microgels behavior. This estimate is consistent with scaling arguments of the Hertzian model as a function of particle size $\mathrm{s}^{51,52}$ with respect to the large microgels. 
Numerical calculation of the effective potential. Microgel configurations are built as disordered, fully-bonded networks generated as in ref. ${ }^{8,40}$ using $\approx 5000$ monomers of diameter $\sigma_{\mathrm{m}}$ in a spherical confinement of radius $Z=25 \sigma_{\mathrm{m}}$ and crosslinker concentration $c=5 \%$. Monomers are in the swollen regime and interact through the classical bead-spring model for polymers ${ }^{53}$.

To calculate the microgel-microgel effective interactions we combine two methods, as described in ref. ${ }^{40}$. We perform umbrella sampling simulations in which we add an harmonic biasing potential acting on the centres of mass of the two microgels ${ }^{54}$ for small separation distances $r$. The resulting effective potential is calculated as $V(r)=$ $-k_{\mathrm{B}} T \ln [g(r)]$. At larger values of $r$, we employ a generalized Widom insertion scheme $\mathrm{e}^{55}$ which is very efficient in sampling the small-deformation regime.

Bulk simulations. We perform Langevin dynamics simulations of $N=2000$ colloid particles of mass $m$ interacting with the generated effective interaction potential $V_{\text {eff,cc. }}$ The units of length, energy, and mass are $\sigma_{\text {eff }}, k_{\mathrm{B}} T$ and $m$ respectively. Time is measured in units of $\sqrt{m \sigma_{\text {eff }}^{2} / k_{\mathrm{B}} T}$. The integration time-step is fixed to 0.001 . With this scheme, particles after an initial microscopic time follow a Brownian Dynamics (BD) due to the interactions with a fictitious solvent ${ }^{56}$. The solvent effective viscosity enters in the definition of the zero-colloid limit self-diffusion coefficient $D_{0}$, which is the key parameter in BD simulations.

Since the viscosity of the real samples changes for each $T$ and for each $\phi_{\text {eff,d }}$, we have estimated the experimental $D_{0}$ at $\phi_{\mathrm{eff}, \mathrm{d}}=0$ by means of Stokes-Einstein relations using the measured hydrodynamic radii at each $T$. Furthermore, we have also estimated $D_{0}$ in the presence of depletant thanks to the viscosity measurements described above. Ideally, one would need to directly use these values in the simulations, but this leads to an incorrect description of the system at low enough colloid packing fractions because BD simulations do not include hydrodynamic interactions, whose effects are strongest in this regime. Hence we have adopted the following strategy: at fixed $\phi_{\text {eff,c }}$ and for each $T$ and $\phi_{\text {eff,d }}$ (i.e., for 16 of our samples), we performed several BD runs in order to select the values of $D_{0}$ providing a good agreement for the long-time MSD with experiments. We thus find a unique shift factor on the time axis for all simulated state points, needed in order to convert simulation time into experimental time. Then, the estimated $D_{0}$ values were kept fixed for all studied $\phi_{\text {eff,c }}$, i.e., for the remaining 32 studied samples no further adjustment was made. The estimated $D_{0}$ can thus be considered the effective bare self-diffusion coefficients of our approximated BD approach, and was finally compared to the experimental estimates, finding a good agreement at large depletant concentrations and low temperatures (as reported in Supplementary Fig. 9 and Supplementary Note 9), that is, for the state points where hydrodynamic interactions are less important.

Simulations were performed with particles possessing a polydispersity of $4 \%$ with a Gaussian distribution, similar to the experimental system. Slices through configurations of 100 independent state points were used to calculate the radial distribution function $g(r)$ of the 3D data with sufficient statistics. The $z$-position of particles is randomly displaced by Gaussian noise with a standard deviation of 0.005 . In this way, $g(r)$ s can be successfully compared to the $2 \mathrm{D}-g(r)$ obtained from experiments, as demonstrated in ref. ${ }^{33}$.

Code availability. The computer codes used for the current study are available from the corresponding authors on reasonable request.

\section{Data availability}

The authors declare that all data supporting the findings of this study are available within the article and its Supplementary Information files. All other relevant data supporting the findings of this study are available from the corresponding authors on request.

Received: 18 January 2018 Accepted: 30 October 2018

Published online: 28 November 2018

\section{References}

1. Vlassopoulos, D. \& Cloitre, M. Tunable rheology of dense soft deformable colloids. Curr. Opin. Colloid In. 19, 561-574 (2014).

2. van der Scheer, P., van de Laar, T., van der Gucht, J., Vlassopoulos, D. \& Sprakel, J. Fragility and strength in nanoparticle glasses. ACS Nano 11, 6755-6763 (2017).

3. Conley, G. M., Aebischer, P., Nöjd, S., Schurtenberger, P. \& Scheffold, F. Jamming and overpacking fuzzy microgels: deformation, interpenetration, and compression. Sci. Adv. 3, el700969 (2017).

4. Mohanty, P. S. et al. Interpenetration of polymeric microgels at ultrahigh densities. Sci. Rep. 7, 1487 (2017).

5. Stieger, M., Pedersen, J. S., Lindner, P. \& Richtering, W. Are thermoresponsive microgels model systems for concentrated colloidal suspensions? a rheology and small-angle neutron scattering study. Langmuir 20, 7283-7292 (2004).
6. Rey, M. et al. Isostructural solid-solid phase transition in monolayers of soft core-shell particles at fluid interfaces: structure and mechanics. Soft Matter 12, 3545-3557 (2016).

7. Boon, N. \& Schurtenberger, P. Swelling of micro-hydrogels with a crosslinker gradient. Phys. Chem. Chem. Phys. 19, 23740-23746 (2017).

8. Gnan, N., Rovigatti, L., Bergman, M. \& Zaccarelli, E. In silico synthesis of microgel particles. Macromolecules 50, 8777-8786 (2017).

9. Mohanty, P. et al. Dielectric spectroscopy of ionic microgel suspensions. Soft Matter 12, 9705-9727 (2016).

10. Hashmi, S. M. \& Dufresne, E. R. Mechanical properties of individual microgel particles through the deswelling transition. Soft Matter 5, 3682-3688 (2009).

11. Bachman, H. et al. Ultrasoft, highly deformable microgels. Soft Matter 11, 2018-2028 (2015).

12. Reese, C. E., Mikhonin, A. V., Kamenjicki, M., Tikhonov, A. \& Asher, S. A. Nanogel nanosecond photonic crystal optical switching. J. Am. Chem. Soc. 126, 1493-1496 (2004).

13. Serpe, M. J. et al. Hydrogel Micro and Nanoparticles, chap. (Wiley-VCH Verlag $\mathrm{GmbH}$ and Co., KGaA, 2012; 317-336. Color-Tunable Poly (NIsopropylacrylamide) Microgel-Based Etalons: Fabrication, Characterization, and Applications.

14. Hamidi, M., Azadi, A. \& Rafiei, P. Hydrogel nanoparticles in drug delivery. Adv. Drug Deliv. Rev. 60, 1638-1649 (2008).

15. Peppas, N., Bures, P., Leobandung, W. \& Ichikawa, H. Hydrogels in pharmaceutical formulations. Eur. J. Pharm. Biopharm. 50, 27-46 (2000).

16. Oh, J. K., Drumright, R., Siegwart, D. J. \& Matyjaszewski, K. The development of microgels/nanogels for drug delivery applications. Prog. Polym. Sci. 33, 448-477 (2008).

17. Fernández-Barbero, A. et al. Gels and microgels for nanotechnological applications. Adv. Colloid Interfac. 147, 88-108 (2009).

18. Wang, Z., Wang, F., Peng, Y., Zheng, Z. \& Han, Y. Imaging the homogeneous nucleation during the melting of superheated colloidal crystals. Science 338, 87-90 (2012).

19. Hilhorst, J. \& Petukhov, A. Variable dislocation widths in colloidal crystals of soft thermosensitive spheres. Phys. Rev. Lett. 107, 095501 (2011).

20. Peng, Y., Wang, Z., Alsayed, A. M., Yodh, A. G. \& Han, Y. Melting of colloidal crystal films. Phys. Rev. Lett. 104, 205703 (2010).

21. Alsayed, A. M., Islam, M. F., Zhang, J., Collings, P. J. \& Yodh, A. G. Premelting at defects within bulk colloidal crystals. Science 309, 1207-1210 (2005).

22. Mohanty, P. S., Bagheri, P., Nöjd, S., Yethiraj, A. \& Schurtenberger, P. Multiple path-dependent routes for phase-transition kinetics in thermoresponsive and field-responsive ultrasoft colloids. Phys. Rev. X 5, 011030 (2015).

23. Zhang, $Z$. et al. Thermal vestige of the zero-temperature jamming transition. Nature 459, 230-233 (2009).

24. Caswell, T. A., Zhang, Z., Gardel, M. L. \& Nagel, S. R. Observation and characterization of the vestige of the jamming transition in a thermal threedimensional system. Phys. Rev. E 87, 012303 (2013).

25. Yunker, P. J. et al. Physics in ordered and disordered colloidal matter composed of poly (n-isopropylacrylamide) microgel particles. Rep. Prog. Phys. 77, 056601 (2014).

26. Heskins, M. \& Guillet, J. E. Solution properties of poly (nisopropylacrylamide). J. Macromol. Sci. Chem. 2, 1441-1455 (1968).

27. Pelton, R. Temperature-sensitive aqueous microgels. Adv. Colloid Interfac. 85, 1-33 (2000)

28. Pelton, R. \& Chibante, P. Preparation of aqueous latices with nisopropylacrylamide. Colloid Surf. 20, 247-256 (1986).

29. Romeo, G., Fernandez-Nieves, A., Wyss, H. M., Acierno, D. \& Weitz, D. A. Temperature-controlled transitions between glass, liquid, and gel states in dense p-nipa suspensions. Adv. Mater. 22, 3441-3445 (2010).

30. Heyes, D. \& Brańka, A. Interactions between microgel particles. Soft Matter $\mathbf{5}$, 2681-2685 (2009).

31. Senff, H. \& Richtering, W. Temperature sensitive microgel suspensions: Colloidal phase behavior and rheology of soft spheres. J. Chem. Phys. 111, 1705-1711 (1999)

32. Wu, J., Huang, G. \& Hu, Z. Interparticle potential and the phase behavior of temperature-sensitive microgel dispersions. Macromolecules 36, 440-448 (2003).

33. Mohanty, P. S., Paloli, D., Crassous, J. J., Zaccarelli, E. \& Schurtenberger, P. Effective interactions between soft-repulsive colloids: Experiments, theory, and simulations. J. Chem. Phys. 140, 094901 (2014).

34. Scheffold, F. et al. Brushlike interactions between thermoresponsive microgel particles. Phys. Rev. Lett. 104, 128304 (2010).

35. Romeo, G. \& Ciamarra, M. P. Elasticity of compressed microgel suspensions. Soft Matter 9, 5401-5406 (2013).

36. Paloli, D., Mohanty, P. S., Crassous, J. J., Zaccarelli, E. \& Schurtenberger, P. Fluid-solid transitions in soft-repulsive colloids. Soft Matter 9, 3000-3004 (2013). 
37. Likos, C. N. Effective interactions in soft condensed matter physics. Phys. Rep. 348, 267-439 (2001).

38. Bayliss, K., Van Duijneveldt, J., Faers, M. \& Vermeer, A. Comparing colloidal phase separation induced by linear polymer and by microgel particles. Soft Matter 7, 10345-10352 (2011).

39. Rovigatti, L., Gnan, N., Parola, A. \& Zaccarelli, E. How soft repulsion enhances the depletion mechanism. Soft Matter 11, 692-700 (2015).

40. Rovigatti, L., Gnan, N., Ninarello, A. \& Zaccarelli, E. On the validity of the hertzian model: the case of soft colloids. Prepr. arXiv 1808.04769 (2018).

41. Hoffmann, N., Ebert, F., Likos, C. N., Löwen, H. \& Maret, G. Partial clustering in binary two-dimensional colloidal suspensions. Phys. Rev. Lett. 97, 078301 (2006).

42. Angioletti-Uberti, S., Varilly, P., Mognetti, B. M. \& Frenkel, D. Mobile linkers on dna-coated colloids: valency without patches. Phys. Rev. Lett. 113, 128303 (2014).

43. Truzzolillo, D. et al. Overcharging and reentrant condensation of thermoresponsive ionic microgels. Soft Matter 14, 4110-4125 (2018).

44. Acciaro, R., Gilányi, T. \& Varga, I. Preparation of monodisperse poly(nisopropylacrylamide) microgel particles with homogenous cross-link density distribution. Langmuir 27, 7917-7925 (2011).

45. Tiwari, R. et al. A versatile synthesis platform to prepare uniform, highly functional microgels via click-type functionalization of latex particles. Macromolecules 47, 2257-2267 (2014).

46. Wei, J., Li, Y. \& Ngai, T. Tailor-made microgel particles: Synthesis and characterization. Colloid Surf. A 489, 122-127 (2016).

47. Mueller, E. et al. Dynamically cross-linked self-assembled thermoresponsive microgels with homogeneous internal structures. Langmuir 34, 1601-1612 (2018).

48. Batchelor, G. The effect of brownian motion on the bulk stress in a suspension of spherical particles. J. Fluid. Mech. 83, 97-117 (1977)

49. Crocker, J. C. \& Grier, D. G. Methods of digital video microscopy for colloidal studies. J. Colloid Interf. Sci. 179, 298-310 (1996).

50. Parola, A. \& Reatto, L. Depletion interaction between spheres of unequal size and demixing in binary mixtures of colloids. Mol. Phys. 113, 2571-2582 (2015).

51. Landau, L. D. \& Lifshitz, E. M. Theory of Elasticity V. 7 of Course of Theoretical Physics (Pergamon Press, Oxford, 165 pages, 1959).

52. Riest, J., Mohanty, P., Schurtenberger, P. \& Likos, C. N. Coarse-graining of ionic microgels: Theory and experiment. Z. Phys. Chem. 226, 711-735 (2012).

53. Grest, G. S. \& Kremer, K. Molecular dynamics simulation for polymers in the presence of a heat bath. Phys. Rev. A. 33, 3628 (1986).

54. Roux, B. The calculation of the potential of mean force using computer simulations. Comput. Phys. Commun. 91, 275-282 (1995).

55. Mladek, B. M. \& Frenkel, D. Pair interactions between complex mesoscopic particles from widom's particle-insertion method. Soft Matter 7, 1450-1455 (2011).

56. Allen, M. P. \& Tildesley, D. J. Computer simulation of liquids (Oxford University Press, Oxford, 640 pages, 2017).

\section{Acknowledgements}

We thank Sofi Nöjd for particle synthesis and Andrea Ninarello for discussions. M.B. and P.S. acknowledge financial support from the European Research Council (ERC-339678COMPASS) and the Swedish Research Council (VR 2015-05426). N.G., L.R. and E.Z. acknowledge support from the European Research Council (ERC Consolidator Grant 681597, MIMIC).

\section{Author contributions}

E.Z. and P.S. designed and supervised research. M.B. performed all experiments with help from M.O.R. and J.M.M. M.B., N.G., L.R., and E.Z. performed simulations and modeling. All authors contributed to the interpretation and analysis of the data. M.B., L.R., E.Z., and P.S. wrote the manuscript with inputs from all other authors.

\section{Additional information}

Supplementary Information accompanies this paper at https://doi.org/10.1038/s41467 018-07332-5.

Competing interests: The authors declare no competing interests.

Reprints and permission information is available online at http://npg.nature.com/ reprintsandpermissions/

Publisher's note: Springer Nature remains neutral with regard to jurisdictional claims in published maps and institutional affiliations.

\begin{abstract}
(c) (i) Open Access This article is licensed under a Creative Commons Attribution 4.0 International License, which permits use, sharing, adaptation, distribution and reproduction in any medium or format, as long as you give appropriate credit to the original author(s) and the source, provide a link to the Creative Commons license, and indicate if changes were made. The images or other third party material in this article are included in the article's Creative Commons license, unless indicated otherwise in a credit line to the material. If material is not included in the article's Creative Commons license and your intended use is not permitted by statutory regulation or exceeds the permitted use, you will need to obtain permission directly from the copyright holder. To view a copy of this license, visit http://creativecommons.org/ licenses/by/4.0/.
\end{abstract}

(C) The Author(s) 2018 